\title{
Low Density Spreading Multiple Access
}

\author{
Mohammed Al-Imari, Muhammad Ali Imran* and Rahim Tafazolli \\ Centre for Communication Systems Research, University of Surrey, Guildford GU2 7XH, Surrey, UK
}

\section{Introduction}

Multiple access (MA) technique is a major building block of the cellular systems. Through the MA technique, the users can simultaneously access the physical medium and share the finite resources of the system, such as spectrum, time and power. Due to the rapid growth in demand on data applications in mobile communications, there has been extensive research to improve the efficiency of cellular systems. A significant part of this effort focuses on developing and optimizing the MA techniques. As a result, many MA techniques have been proposed systematically over the years, and some of these MA techniques are already been adopted in the cellular system standards such as Time Division Multiple Access (TDMA), Frequency Division Multiple Access (FDMA), Orthogonal Frequency Division Multiple Access (OFDMA) and Code Division Multiple Access (CDMA). There are many factors that determine the efficiency of the MA technique such as spectral efficiency, low complexity implementation as well low envelope fluctuations.

Mainly, the MA techniques can be categorized into orthogonal and non-orthogonal MA. In orthogonal MA techniques, the signal dimension is partitioned and allocated exclusively to the users, and there is no Multiple Access Interference (MAI). For non-orthogonal MA techniques, all the users share the entire signal dimension, and there is a MAI. Thus, for non-orthogonal transmission, more complicated receiver is required to deal with the MAI comparing to orthogonal transmission. Non-orthogonal MA is more practical in the uplink scenario because the base station can afford the Multiuser Detection (MUD) complexity. On the other hand, for downlink, orthogonal MA is more suitable due to the limited processing power at the user equipment. Many non-orthogonal MA techniques have been overlooked due to the implementation complexity. Evidently, the recent advancements in signal processing have opened up new possibilities for developing more sophisticated and efficient MA techniques. Thus, more advanced MA techniques has been proposed lately. However, in order to adopt these new MA techniques in the mobile communication systems, many challenges and opportunities need to be studied.

\section{Low Density Spreading}

One of these promising non-orthogonal MA techniques is the Multicarrier-Low Density Spreading Multiple Access (MC-LDSMA) technique [1-3]. In MC-LDSMA, Low Density Spreading (LDS) codes are used for transmission, where each user spreads its data on a small set of subcarriers. There is no exclusivity in the subcarriers allocation and more than one user can share each subcarrier. The interference pattern at the receiver will generate a low density graph, and graph theory based techniques can be utilized [4]. The main features of the LDS scheme can be summarized as follow [1]:

- At each subcarrier, a user will have relativity small number of interferers comparing to the total number of users. Consequently, the search space will be smaller and more complex MUD techniques can be implemented.

- Higher Signal to Interference plus Noise Ratio (SINR) can be achieved at each subcarrier, which results in reliable detection process.
- Each user will experience interference from different users at different subcarriers, which results in interference diversity by avoiding strong interferers to destroy the signal of a user on all the subcarriers.

- Belief propagation based MUD can be implemented with linear complexity in the number of subcarriers [4].

Accordingly, benefiting from these features, MC-LDSMA demonstrated a remarkable link-level performance, in terms of Bit Error Rate (BER), comparing to many exist MA techniques [1-3]. Hence, the transmission power required to achieve specific Qualityof-Service (QoS) is less comparing to another MA techniques, which means longer battery life and more cell coverage $[2,3]$.

Unlike conventional spreading schemes such as MC-CDMA, there are no auto-correlation or cross-correlation constraints on the LDS codes. In fact, random LDS codes have shown to achieve BER close to the single-user bound [1]. Consequently, there is a big room to be exploited in the LDS codes design. This flexibility includes the LDS codes' phases, subcarrier allocation, and power allocation. For instance, the MC-LDSMA inherits the high envelope fluctuations drawback of multicarrier communications, which is more critical for the uplink case due to the limited power of the user equipment. The LDS codes' phases can be optimized to reduce the envelope fluctuations. Using simple yet efficient phasing scheme, the envelope fluctuations of MC-LDSMA signals are significantly reduced $[2,3,5]$.

The aforementioned advantages of MC-LDSMA are mainly from the link-level perspective, which were the main motivations for the design of the LDS technique [1,4]. However, there is another advantage of MC-LDSMA from the system-level perspective that can be exploited to improve the system performance. MC-LDSMA offers an opportunity to dynamically allocating the subcarrier and power without affecting the receiver performance [6]. Due to the limitations of radio resources such as spectrum and power, optimal utilization of these scarce resources is essential in cellular systems. Radio resource allocation can plays a key role in optimizing the performance of $\mathrm{MC}$ LDSMA system by exploiting the frequency and multi-user diversity gains. One important metric in radio resources allocation is the users' sum-rate. However, using sum-rate as optimization criterion is not fair for users with bad channel conditions, i.e. cell-edge users. Considering fairness in orthogonal MA, such as OFDMA, reduces the user' sumrate because the subcarriers that allocated to cell-edge users are not available for users close to the base station. On the other hand, in MC-LDSMA technique there is no such constraint and the subcarrier

*Corresponding author: Muhammad Ali Imran, Centre for Communication Systems Research, University of Surrey, Guildford GU2 7XH, Surrey, U.K, Tel: 44-1483-686003; Fax 44-1483-686011; E-mail: m.imran@surrey.ac.uk

Received September 17, 2012; Accepted September 21, 2012; Published September 24, 2012

Citation: Al-Imari M, Imran MA, Tafazolli R (2012) Low Density Spreading Multiple Access. J Inform Tech Softw Eng 2:e108. doi:10.4172/2165-7866.1000e108

Copyright: @ $2012 \mathrm{Al}-$ Imari M, et al. This is an open-access article distributed under the terms of the Creative Commons Attribution License, which permits unrestricted use, distribution, and reproduction in any medium, provided the original author and source are credited. 
can be shared by more than one user in the same time. Thus, the subcarriers that been allocated to cell-edge users to achieve fairness can be used by users with good channel conditions to improve the system spectral efficiency. In fact, from the information theory perspective, the only approach to achieve fairness among the users is by using nonorthogonal MA techniques [7].

With subcarrier and power allocation, MC-LDSMA can significantly outperform current MA techniques in terms of spectral efficiency and fairness [2,3,6]. Even though the LDS structure imposes constraints on the number of users share the same subcarrier, the system performance can still be significantly improved by using MCLDSMA technique. The spectral efficiency and fairness among users are increased by allowing more users share the subcarrier. However, high number of users per subcarrier is not necessary and satisfactory performance can be achieved with small number of users per subcarrier $[2,3]$. These gains in the system-level performance can be translated into an increase in cell coverage, users supported in the cell and users' data rates.

\section{Conclusion}

The growing demand for efficient and high data-rate applications in cellular systems has attracted significant research attention to develop more efficient multiple access techniques. Many open problems remaining in the field that need to be tackled and open access journals can accelerate the progress in this research area by making the state of art developments freely available for all researchers. OMICS group of publications is one of the suitable places to share new research findings and make significant impact of ideas and solutions.

\section{References}

1. Wathan $F$ (2007) Low-density signature communications in overloaded CDMA systems.

2. Al-Imari M, Imran MA, Tafazolli R (2012) Low Density Spreading for next generation multicarrier cellular systems. International Conference on Future Communication Networks (ICFCN) 52-57.

3. Al-Imari M, Imran MA, Tafazolli R, Chen D (2012) Performance Evaluation of Low Density Spreading Multiple Access. International Wireless Communications and Mobile Computing Conference (IWCMC), 27-31.

4. Montanari A, Tse D (2006) Analysis of belief propagation for non-linear problems: The example of CDMA (or: How to prove tanaka's formula). 160 164

5. Al-Imari M, Hoshyar R (2010) Reducing the Peak to Average Power Ratio of LDS-OFDM signals. 7th International Symposium on Wireless Communication Systems (ISWCS) 922-926

6. Al-Imari M, Imran MA, Tafazolli R, Chen D (2011) Subcarrier and Power Allocation for LDS-OFDM System. IEEE 73rd Vehicular Technology Conference (VTC spring) 1-5.

7. Tse DNC, Hanly SV (1998) Multiaccess fading channels-Part I: Polymatroid structure, optimal resource allocation and throughput capacities. IEEE Transactions on Information Theory 44: 2796-2815.
Submit your next manuscript and get advantages of OMICS Group submissions

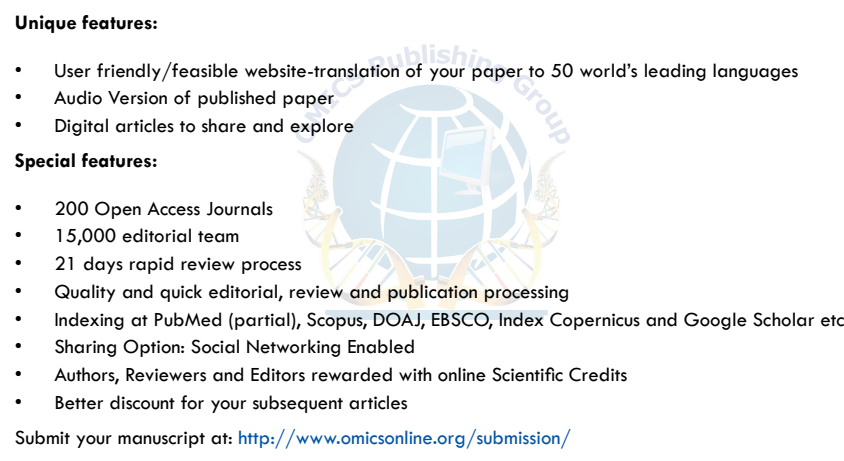

